We believe that restoration of adequate coaptation at the annular level is essential for a competent valve. Saline injection to the left ventricle may not represent the systolic phase of the mitral valve, and thus the length of artificial chordae determined by this measurement may not be correct.

Preoperative measurement of chordae by transesophageal echocardiography has an advantage in that it reduces crossclamp and bypass times. ${ }^{2}$ It requires an experienced operator, however, to avoid tilting the measurement and thus achieve a correct length. Also, the points at which the needle passes through the papillary muscle and leaflet may be different from the points of measurement. That changes the desired length.

In our experience, use of the corresponding nonprolapsed chordal length is the best method for tailoring artificial chordae. Intraoperative measurement of the length of nonprolapsed segment with a caliper has been proposed by others. ${ }^{4}$ Our method represents a similar idea but allows us to skip the process of measuring and then making artificial chordae outside the operative field. The decrease in operative steps reduces operative time and thus potential for errors.

The easiest way to fix a knot is to tie it on a fixed point. Because Gore-Tex is a monofilament suture, crushing should be avoided to prevent weakening its tensile strength, as specified in the manufacturer's instructions. Although double-sided rubber-protected artery forceps can prevent crushing, the suture may slip while tying knots. Since adopting a single-sided rubber-protected clamping technique, we have not encountered Gore-Tex suture rupture or knot slippage. Moreover, only 4 of 87 artificial chordae (4.6\%) have required revision for incorrect length. This means that most measurements with this technique are accurate. The instruments used in this method are easily available and inexpensive (Figure 1, $F$ ).

This straightforward and reproducible technique makes the use of artificial chordae an effective and simple method to treat leaflet prolapses in mitral regurgitation.

\section{References}

1. David TE, Omran A, Armstrong S, Sun Z, Ivanov J. Long-term results of mitral valve repair for myxomatous disease with and without chordal replacement with expanded polytetrafluoroethylene sutures. J Thorac Cardiovasc Surg. 1998;115: 1279-86.

2. Mandegar MH, Yousefnia MA, Roshanali F. Preoperative determination of artificial chordae length. Ann Thorac Surg. 2007;84:680-2.

3. Maselli D, Paulis D, Weltert L, Salica A, Scaffa R, Bellisario A, et al. A new method for artificial chordae length "tuning"' in mitral valve repair: preliminary experience. J Thorac Cardiovasc Surg. 2007;134:454-9.

4. Tam R, Joshi P, Konstantinov IE. A simple method of preparing artificial chordae for mitral valve repair. J Thorac Cardiovasc Surg. 2006;132:1486-7.

\title{
Reversed L-shaped deformity of the anterior mitral leaflet and its reparative technique
}

\author{
Shinji Masuyama, MD, ${ }^{*}$ Akira Marui, MD, PhD, ${ }^{*}$ Takeshi Shimamoto, MD, and Masashi Komeda, MD, PhD, Kyoto, Japan
}

Although mitral valve repair for mitral regurgitation (MR) has become very popular, surgeons sometimes encounter difficult cases to repair. In this article we report 2 cases of successful repair for complex reversed L-shaped deformity, a complex lesion of anterior mitral leaflet (AML) prolapse (ie, toward the left atrium) with tethering (ie, toward the left ventricle), the former by elongation or rupture of the

\footnotetext{
From the Department of Cardiovascular Surgery, Graduate School of Medicine, Kyoto University, Kyoto, Japan

* Both authors equally contributed to this work.

Received for publication Dec 1, 2007; accepted for publication Dec 9, 2007.

Address for reprints: Masashi Komeda, MD, PhD, Department of Cardiovascular Surgery, Graduate School of Medicine, Kyoto University, 54 Shogoin-Kawara, Sakyo, Kyoto 606-8507, Japan (E-mail: komelab@kuhp.kyoto-u.ac.jp).

J Thorac Cardiovasc Surg 2008;136:1599-601

$0022-5223 / \$ 34.00$

Copyright (c) 2008 by The American Association for Thoracic Surgery

doi:10.1016/j.jtcvs.2007.12.051
}

primary chordae and the latter by tethering of the secondary chordae (Figure 1).

\section{CLINICAL SUMMARY}

Surgical treatment for reversed L-shaped deformity is a combination of the following 3 techniques: cutting of the secondary chordae, ${ }^{1}$ translocation of the secondary chor$\mathrm{dae}^{2}$ and reconstruction of the primary chordae ${ }^{3}$ for the AML (Figure 2).

After median sternotomy and during cardiopulmonary bypass with cardiac arrest, the mitral valve was exposed through a standard right-sided left atriotomy.

First, secondary chordal cutting is performed. Secondary chordae are recognized by flipping over the anterior leaflet. In this chordal cutting step, all the secondary chordae were severed at their insertion to the AML for ameliorating the leaflet tethering. 

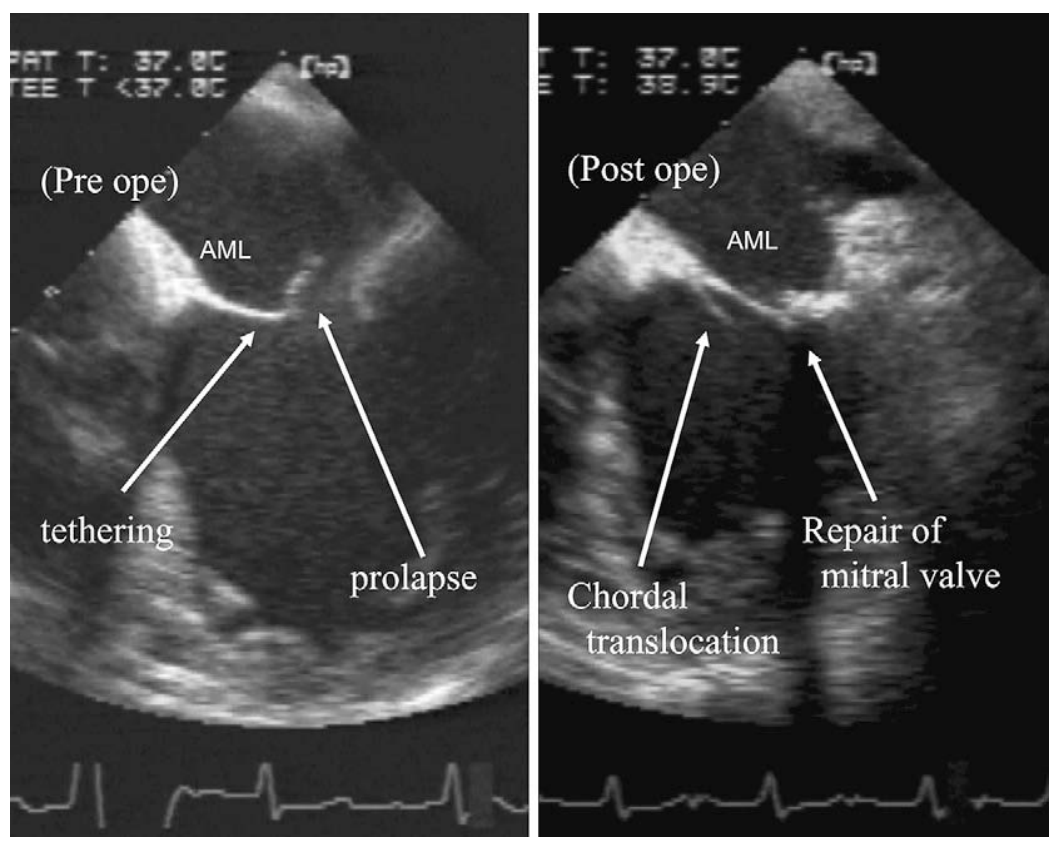

FIGURE 1. Representative pre- and post- operative echocardiographic images of "Reversed L-shaped" deformity. Pre ope, Preoperative; (Preop) simultaneous existance of prolapse and tethering of the AML deformed it "Reversed L-shape". Post ope, postoperative; (Postop) Both prolapse and tethering of the AML disappeared. $A M L$, anterior mitral leaflet.

Second, chordal translocation is performed, wherein a Teflon-pledgeted 5-0 polytetrafluoroethylene (PTFE) suture was placed in each papillary muscle tip, which is the site of origin of the secondary chordae. The suture is then passed through the midseptal annulus (saddle horn) and through the left atrial wall and then put through the tourniquet and cross-clamped to fix the chordal length at the desired chordal tension. Care is taken to avoid damaging the aortic cusps. The length of this chordal translocation stitch is adjusted such that it is taut to avoid excess tension on the chordae and it maintains valvular-ventricular continuity and left ventricular (LV) dimension/function. ${ }^{2}$

Finally, AML prolapse is repaired by using the conventional chordal reconstruction technique. A Teflon-pledgeted 5-0 PTFE suture, which is used as artificial chordae, is placed in the posterior papillary muscle tip. The PTFE chordae are brought through the free edge of elongation or the ruptured segment and fixed with adjusted length. In both cases annuloplasty was performed: a flexible ring was sutured to the mitral annulus with 2-0 Tycron interrupted mattress sutures.

We experienced 2 cases of reverse L-shaped deformity of the AML and successfully treated them with our surgical strategy. Case 1 was a 75 -year-old woman who presented with severe MR and accompanying LV dysfunction (left ventricular ejection fraction [LVEF], 19\%) because of dilated cardiomyopathy. Echocardiography revealed (1) AML prolapse caused by primary chordal rupture in the middle to posterolateral side of the AML and (2) tethering (depth of tethering, $5 \mathrm{~mm}$ ) of the AML. Case 2 was a 64year-old man with ischemic cardiomyopathy (LVEF, $19 \%$ ) and moderate MR. Echocardiographic analysis revealed (1) prolapse of the AML caused by elongation of the primary chordae and (2) severe tethering (depth of tethering, $9 \mathrm{~mm}$ ) of the AML. In both patients the postoperative courses were uneventful, and echocardiographic analysis showed the absence of residual MR and tethering and improvement in LVEF $(30 \%$ and $60 \%$ in cases 1 and 2 ,

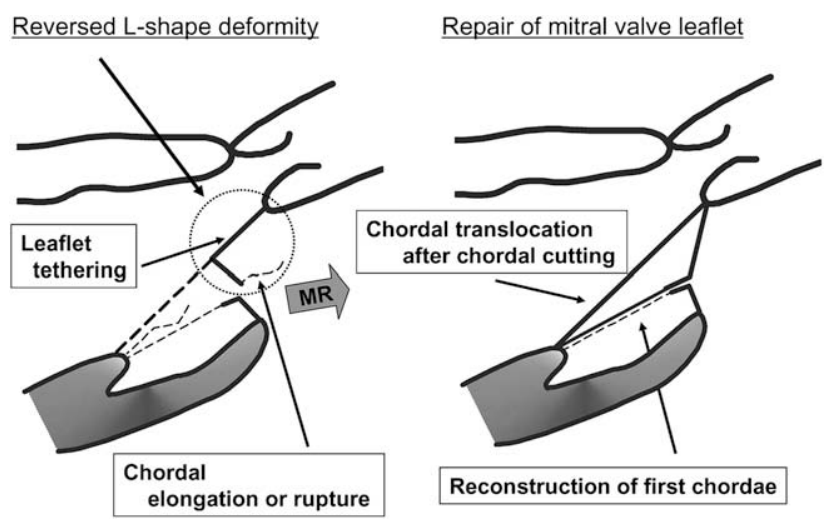

FIGURE 2. Repair of the reversed L-shape deformity. Cutting the secondary chordae, translocation of the secondary chordae, and reconstruction of the primary chordae for the AML are necessary to repair the deformity. $M R$, mitral regurgitation. 
respectively). No cardiac event occurred during the followup period (24 and 25 months in cases 1 and 2, respectively).

\section{DISCUSSION}

In this report we have described 2 surgical cases of complex MR with reversed L-shaped deformity of the AML and have discussed the repair technique for the lesion. However, the treatment of AML prolapse is more complex than that of posterior mitral leaflet (PML) prolapse, and it does not necessarily provide a satisfactory long-term result.

Several techniques, such as chordal transfer, ${ }^{4}$ papillary muscle repositioning, ${ }^{5}$ and chordal reconstruction, ${ }^{3}$ have been reported for the repair of AML prolapse. Chordal transfer (PML to AML) is an excellent technique to treat AML prolapse but is limited by the number of chordae available. In addition, it is not ideal that PML be made use of for the repair of AML prolapse because the PML is intact and the procedure can create new "disease" to the intact part of the PML. It should be avoided if possible. Papillary muscle repositioning is also an effective technique for the repair of AML prolapse. However, if the prolapse is accompanied by leaflet tethering, papillary muscle repositioning can lead to further deterioration of leaflet tethering.

Chordal reconstruction ${ }^{3}$ is advantageous for the treatment of AML prolapse. This technique does not require leaflet resection and is useful for the repair of any type of prolapse. In our case, because there were no degenerative changes in the AML, we conjectured that leaflet resection could be avoided. However, artificial chordal replacement alone could not resolve the problem of residual tethering. Residual tethering might lead to recurrent functional regurgitation during the postoperative period.

To overcome the limitations of chordal cutting, we have developed a chordal translocation stitch that restores the continuity between the papillary muscle tips and the anterior mitral annulus. Chordal cutting, ${ }^{1}$ which involves the disruption of the secondary chordae, is a simple method performed to repair tethering. However, to accomplish chordal cutting, the first chordae should be intact. If chordal cutting is performed with ruptured or elongated first chordae, MR can deteriorate because of worsening AML prolapse. Hence care must be taken to detect lesions in the first chordae before performing chordal cutting. Another limitation of chordal cutting is that it involves the excision of secondary chordae that maintain mitral valvular-ventricular continuity, which plays an important role in LV structure and function. Thus chordal cutting might reduce AML tethering and mitral valve regurgitation at the cost of LV systolic geometry, function, or both. A combination of chordal placement and chordal cutting/translocation might be helpful to repair the valve in patients with poor $\mathrm{LV}$ function.

In conclusion, a combination of secondary chordal cutting, chordal translocation, and chordal reconstruction of the AML can be effective to treat reversed L-shaped deformity, which is a complex AML lesion. Further studies are warranted in clinical settings.

\section{References}

1. Messas E, Guerrero JL, Handschumacher MD, Conrad C, Chow CM, Sullivan S, et al. Chordal cutting: a new therapeutic approach for ischemic mitral regurgitation. Circulation. 2001;104:1958-63.

2. Fukuoka M, Nonaka M, Masuyama S, Shimamoto T, Tambara K, Yoshida H, et al Chordal "translocation" for functional mitral regurgitation with severe valve tenting: an effort to preserve left ventricular structure and function. J Thorac Cardiovasc Surg. 2007;133:1004-11.

3. Lawrie GM, Earle EA, Earle NR. Feasibility and intermediate term outcome of repair of prolapsing anterior mitral leaflets with artificial chordal replacement in 152 patients. Ann Thorac Surg. 2006;81:849-56.

4. Gillinov AM, Cosgrove DM. Chordal transfer for repair of anterior leaflet prolapse. Semin Thorac Cardiovasc Surg. 2004;16:169-73.

5. Dreyfus GD, Souza Neto O, Aubert S. Papillary muscle repositioning for repair of anterior leaflet prolapse caused by chordal elongation. J Thorac Cardiovasc Surg. 2006;132:578-84. 